\title{
Overview of the
}

\section{ARPA Human Language Technology Workshop}

\author{
Madeleine Bates, Chair, Editor \\ BBN Systems \& Technologies \\ 70 Fawcett Street \\ Cambridge, MA 02138
}

\section{PREVIOUS DARPA WORKSHOPS}

For five years, 1988-1992, the Defense Advanced Projects Agency sponsored a series of meetings called the DARPA Speech and Natural Language Workshops. These workshops provided a forum where researchers in speech and natural language, particularly as relating to the DARPA programs in spoken and written language understanding, could exchange information about recent research and technical progress.

Participants included researchers funded under the DARPA programs, other researchers who voluntarily participated in these programs or in related evaluations, government researchers and consumers of these research results, and invited attendees from inside and outside the US.

Proceedings of these workshops were published by Morgan Kaufmann.

\section{THE 1993 WORKSHOP: EXPANDED SCOPE}

In 1993, the " $D$ " was dropped from DARPA, reflecting a change in the organizational purposes and goals. Well before this change, however, the committee responsible for the annual workshop was directed to significantly broaden its focus, and the name of the meeting became The ARPA Human Language Technology (HLT) Workshop.

The HLT workshop provides a forum where researchers can exchange information about very recent technical progress in an informal, highly interactive setting. The scope includes not just speech recognition, speech understanding, text understanding, and machine translation, but also all spoken and written language work (broadly interpreted to include ARPA's TIPSTER, MT, MUC, and TREC programs) with an emphasis on topics of mutual interest, such as statistical language modeling. This workshop no longer focuses on spoken language systems evaluation, as another workshop fills that need.

The prime purpose of the newly constituted workshop was to facilitate productive technical discussions among key researchers on topics relating to human language technology and of interest to ARPA. This expanded workshop, in par- ticular, was designed to facilitate cross-fertilization among diverse disciplines, and to introduce researchers to the stateof-the-art in areas outside their own. For this reason, the papers presented in this proceedings are both more widereaching and more accessible to non-experts in the field than has previously been the case.

The majority of the workshop participants received funding under ARPA's Human Language program. Other participants included researchers not funded by ARPA who voluntarily participate in these programs; government researchers and consumers of these research results; and, on a rotating basis, selected visitors from both inside and outside the United States. It is still the intention that the participants form a tightly-coupled research community in which results and research breakthroughs are evaluated, disseminated, and exploited with very short latency.

Aspects of this meeting included:

- technical presentations of both new research results and the ongoing development of large software systems, often months before this research is reported elsewhere;

- the presentation of summaries of standardized system evaluations;

- discussion of the future direction of the various DARPA programs in light of recent progress; and

- much discussion of ongoing work among individual researchers.

The format of the meeting was to mix 12-15 minute technical presentations (both reviewed and invited) in sessions organized around focused topics, with time for informal discussion and interaction. In most sessions, the chair presented a 15 minute introduction intended to provide a summary of the key points in each set of papers, and also a perspective of the research context in which the work was done, for those not in the primary area addressed by the papers in the session. The written version of those introductions will serve the same functions in this proceedings. 
Participants selected by the program committee were invited to submit abstracts in a variety of areas. The committee received 131 abstracts (114 for regular presentations; 17 for demonstrations), 58 presentation and 13 demonstrations were accepted.

The final program sessions were: Spoken Language Systems, Invited Overviews of ARPA Program Areas, Continuous Speech Recognition, Natural Language, Discourse, Machine Translation, Demonstrations, Statistical Natural Language, Government Panel, Lexicon, Prosody, Information Retrieval, and New Directions.

Technical highlights included:

- Machine Translation session, chaired by Alex Waibel of CMU. This session featured invited tutorials by Peter Brown and Ed Hovy.

- A demonstration session (chaired by Hy Murveit of SRI and organized by Victor Abrash of SRI) showcased recent work in a variety of areas, and demonstrations were available almost continuously throughout the workshop for "hands-on" experience.

- A lexicon session, chaired by Ralph Grishman (NYU).

- Invited overviews of each of the areas of interest to ARPA in spoken and written language technology: MUC (Beth Sundheim), TREC (Donna Harmon), SLS (George Doddington), and Tipster (Tom Crystal).

- A govemment panel on Govemment Human Language Technology Needs, Funding, In-House Research, and Technology Transfer organized by Carol Van EssDykema. The panelists were Helen Gigley (NRL), Joseph Kielman (FBI), Susan Chipman (ONR), Jesse Fussell (DoD), and Y.T. Chien (NSF).

The workshop attracted 207 attendees, approximately $2 / 3$ from ARPA sites. The remainder were government representatives (30 individuals from 10 different organizations), foreign guests (11 individuals, from 11 organizations), nonARPA attendees from the USA (31 individuals from 27 organizations) and young researchers (7 individuals, from 5 schools).

\section{ACKNOWLEDGEMENTS}

This meeting involved the work of a great many people. The Standing Workshop Committee (whose membership rotates slowly except for one government member) is responsible for the overall series of workshops. It was chaired this year by Patti Price (SRI); other members included Mitch Marcus (U. Penn), Madeleine Bates (BBN), Carol Van Ess-Dykema
(DoD), Cliff Weinstein (Lincoln Lab), and Ralph Grishman (NYU). The standing committee, and Patti Price in particular, provided invaluable direction for the workshop.

The Program Committee, which is constituted each year by adding to the standing committee additional researchers selected for their expertise in specific areas, was chaired by me, and included (in addition to the standing committee members) Stephen Della Pietra, David Lewis, Kathy McKeown, Mary Ellen Okurowski, Stephanie Seneff, Beth Sundheim, and Alex Waibel. The program committee was responsible for reviewing abstracts to select papers and demonstrations for presentation, discussing various policy issues, and organizing the overall program.

Particular thanks are due to Hy Murveit and Victor Abrash, who took complete charge of the demonstration sessions and handled the myriad of technical problems inherent in arranging for a large number of live demos; to Alex Waibel, who organized the Machine Translation session (the keystone session of the entire workshop) while commuting from his office in Germany to his office in the US, and to Ralph Grishman, who organized the Lexicon session apparently effortlessly (which is to say, with extreme competence). Carol Van Ess-Dykema, assisted by Mary Ellen Okurowski, planned and chaired the government panel.

Denise Payne served as the workshop administrator, which means everything from email archivist to registrar. She handled all the interactions with the conference center, kept track of invitations, abstracts, and attendees. She kept one eye on the budget and one hand on the telephone. She prepared the notebooks of preliminary papers that were given to attendees at the workshop, and collected final versions of the papers for this proceedings.

From his new position as Program Manager at ARPA, George Doddington provided overall direction and encouragement to the workshop planners. His enthusiasm for his new role and his desire to continue the high technical standards of previous workshops helped to make this workshop, which was the first in a new series, a resounding success. 